\title{
Non-linear statistical downscaling of present and LGM precipitation and temperatures over Europe
}

\author{
M. Vrac ${ }^{1}$, P. Marbaix ${ }^{2}$, D. Paillard ${ }^{1}$, and P. Naveau ${ }^{1}$ \\ ${ }^{1}$ Laboratoire des Sciences du Climat et de l'Environnement, LSCE,CEA/CNRS/UVSQ, Institut Pierre Simon Laplace (IPSL), \\ Centre d'étude de Saclay, Orme des Merisiers, 91191 Gif-Sur-Yvette, France \\ ${ }^{2}$ Université catholique de Louvain, Institut d'Astronomie et de Géophysique Georges Lematre, 2 chemin du Cyclotron, B348 \\ Louvain-la-Neuve, Belgique
}

Received: 20 June 2007 - Published in Clim. Past Discuss.: 6 July 2007

Revised: 18 October 2007 - Accepted: 27 November 2007 - Published: 19 December 2007

\begin{abstract}
Local-scale climate information is increasingly needed for the study of past, present and future climate changes. In this study we develop a non-linear statistical downscaling method to generate local temperatures and precipitation values from large-scale variables of a Earth System Model of Intermediate Complexity (here CLIMBER). Our statistical downscaling scheme is based on the concept of Generalized Additive Models (GAMs), capturing nonlinearities via non-parametric techniques. Our GAMs are calibrated on the present Western Europe climate. For this region, annual GAMs (i.e. models based on 12 monthly values per location) are fitted by combining two types of largescale explanatory variables: geographical (e.g. topographical information) and physical (i.e. entirely simulated by the CLIMBER model).
\end{abstract}

To evaluate the adequacy of the non-linear transfer functions fitted on the present Western European climate, they are applied to different spatial and temporal large-scale conditions. Local projections for present North America and Northern Europe climates are obtained and compared to local observations. This partially addresses the issue of spatial robustness of our transfer functions by answering the question "does our statistical model remain valid when applied to large-scale climate conditions from a region different from the one used for calibration?". To asses their temporal performances, local projections for the Last Glacial Maximum period are derived and compared to local reconstructions and General Circulation Model outputs.

Our downscaling methodology performs adequately for the Western Europe climate. Concerning the spatial and temporal evaluations, it does not behave as well for Northern America and Northern Europe climates because the calibration domain may be too different from the targeted regions. The physical explanatory variables alone are not capa-

Correspondence to: $\mathrm{M}$. Vrac

(mathieu.vrac@cea.fr) ble of downscaling realistic values. However, the inclusion of geographical-type variables - such as altitude, advective continentality and moutains effect on wind (W-slope) - as GAM explanatory variables clearly improves our local projections.

\section{Introduction}

Understanding the present climate and explaining its changes represent difficult scientific issues. One classical approach to respond to these challenges is to study the past climate history. The class of General Circulation Models (GCM), complex computer codes simulating the atmospheric circulation through resolving the equations representing the Earth's atmospheric dynamics and the coupling between the oceans and the atmosphere, offers a powerful device to understand and represent the global scale behavior of our climate. However, they are computationally intensive and can only produce relatively low spatial resolution simulations. Smallscale physical processes which drive important local surface variables such as heavy precipitation and its strong spatial variability (e.g. Wood et al., 2004) are not yet well captured by GCMs. For paleoclimate studies, this computing time limitation becomes even more prevalent. Only models with a very low resolution are capable of simulating climate over thousand of years (e.g. Rahmstorf and Ganopolski, 1999) and comparing these large-scale outputs with local proxy data corresponds to an arduous task (e.g. Kageyama et al., 2006).

While GCMs aim to capture large-scale behaviors, assessing the regional/local impacts of climate changes constitutes one of the essential inquiries asked by the end-user (economists, decision makers, etc). Depending on the application at hand, the desired time scale can greatly vary. The flood planner may be interested in local precipitation information for the next 50 years and the nuclear waste manager would like to have a rough view of precipitation over his/her

Published by Copernicus Publications on behalf of the European Geosciences Union. 
deposit site for the next thousand years. The latter situation poses more computational and uncertainty problems than the former case. In this paper, we focus on statistical models and large-scale outputs that can span over a few thousands years, present time and the Last Glacial Maximum (LGM). This temporal constraint has been paramount in the data and methods proposed in this paper and, especially it limits the applicability of physical based models that have been devised to handle a few decades or centuries.

The so-called downscaling methods aims at answering how to "zoom in" the information provided by large-scale systems (e.g. GCMs) into the required local-scale. Regional Climate Models (RCMs) provide a physically based approach (e.g. Liang et al., 2006). But, they are generally considered as equally (if not more) computationally intensive than GCMs. The class of Statistical Downscaling Methods (SDMs) represents a well-developped alternative that can bypass this computational obstacle. They are faster because they rely on empirical relationships between local-scale data (e.g. observations, reconstructions) and large-scale upper-air atmospheric variables (e.g. reanalysis data, GCM outputs). Another advantage resides in their probability foundation that allows to associate uncertainties more easily than with RCMs (e.g. Katz, 2002). The SDM proposed in this study belongs to the "transfer functions" SDM family (e.g. Zorita and von Storch, 1998; Snell et al., 2000; Huth, 2002). They directly link large-scale information to local-scale variables. This technique is in contrast to the "weather typing" approach (e.g. Bardossy et al., 1994; Huth, 2001; Vrac et al., 2007a) that is based on conditioning statistical models on recurrent weather states and to weather generators (e.g. Wilks, 1999; Wilks and Wilby, 1999).

One constraining assumption of any SDM resides in the hypothesis that the fitted transfer functions are stationary in time. While this assumption may hold reasonably well at a decadal or even centennial scale, its validity can be strongly challenged over thousands of year. This could lead to unrealistic statistical relationships and then to unrealistic projections of local variables. Consequently, extreme cautions have to be applied when implementing and interpreting a paleoclimatic downscaling scheme. Validity procedures and a search for stationary components have to be added (e.g. Vrac et al., 2007b). For example, incorporating geographical variables that are, by nature, much less sensitive to changing large-scale conditions than physical ones can bring some stability and confidence to paleoclimatic downscaling projections. Another important issue linked to SDMs concerns the statistical modeling type of large-versus-local relationships. Classically, such relationships are assumed to be linear (e.g. Wilby et al., 1998). But, whenever strong nonlinearities are present, these linear models cannot perform adequately. One of our goals in this paper is to present statistical models that have the flexibility of depicting any transfer function (linear and non-linear). They will be non parametric, i.e. the relationship type would not be imposed a priori, but instead, driven by the data themselves.

The rest of this article is organized as follows. In Sect. 2, our large and local-scale data are presented. The proposed statistical model is developed and explained. An application is presented in Sect. 3. Conclusions and a short discussion are provided in the Sect. 4.

\section{Our non-linear statistical downscaling scheme}

To calibrate any SDM, large-scale and local-scale data are required. The local-scale data must have the resolution needed for local projections. The large-scale data resolution has to be equal to the one used to drive the projections.

In this article, the calibration region corresponds to Western Europe and it can approximately be represented by the geographical rectangle $\left[10^{\circ} \mathrm{E} ; 20^{\circ} \mathrm{W}\right] \times\left[37^{\circ} \mathrm{N} ; 55^{\circ} \mathrm{N}\right]($ see maps of Fig. 7). This rectangle has been choosen because it contains various physical and geographical contrasted conditions. Oceanic influences (e.g. Atlantic, North Sea), high mountains near the center (Alps, Pyrenees), Mediterranean conditions, and continental climate (eastern countries) imply a wide range of local temperatures and precipitation values and of large-scale variables. This large variety of climate and geographical situations is necessary to calibrate our transfer functions and to apply them under evolving large-scale conditions.

\subsection{Large-scale data: the CLIMBER model}

Our large-scale data are outputs from the CLIMBER model (Petoukhov et al., 2000, 2005) that has a large spatial resolution of $10^{\circ}$ in latitude and $51^{\circ}$ in longitude. It is an intermediate complexity model. In comparison to 3-D general circulation models, it includes less explicit representations of atmospheric features, thus relying on more parameterizations. In particular, it does not compute explicitly the atmosphere dynamics at the synoptic scales but it accounts for their effects on the meridional heat transport. In addition, the daily variability of meteorological events (e.g. winds associated with low pressure systems) is ignored, as well as the time scale of a few years, in particular the North Atlantic Oscillation.

The CLIMBER model has been conceived to explore climate variations over very long periods $\left(10^{3}\right.$ to $10^{6}$ years $)$. Thus, it is a suitable tool for the paleoclimate community. Despite its simplicity, CLIMBER was favorably compared to results from more complex models, both in a paleoclimate framework (Kageyama et al., 2001) as well as in an upcoming global warming context (Pethoukhov et al., 2005). Nevertheless, it is crucial to keep in mind that CLIMBER outputs largely depend on other components of the climate system, notably on ice sheets and the carbon cycle, that are imposed in CLIMBER. In this work, the outputs are obtained from a control run stabilized after a few thousand years. The 
insolation, $\mathrm{pCO}_{2}$ and ice-sheet forcing are imposed an in Berger (1978), Petit et al. (1999), and Peltier (1994).

\subsection{Local-scale data: the CRU climatology}

The local-scale temperature and precipitation data used for calibration and validation of our statistical model come from the "Climate Research Unit" database (CRU, New et al., 2000). The spatial resolution of these regularly gridded data is high $(10$ ', i.e. $1 / 6$ degree) and it corresponds to more than 13200 land grid-points over our Western Europe region. Their monthly temporal resolution is consistent with the CLIMBER one.

The CRU database represents a monthly present climatology. That means that twelve monthly mean values, representative of the actual climate, are available at each grid-point and for each available variable (here, temperatures and precipitation). In addition, CRU provides the mean altitude of each grid-point.

\subsection{Our statistical method: generalized additive models}

Our goal is here to present a statistical model capable of regressing the values of local variables $Y$ (temperatures or precipitation), called explained variables taken from the CRU database. The explanatory variables $X_{j}$ - i.e. the variables used to explain $Y$ - correspond to some of the large-scale values derived from CLIMBER outputs (see next Section). To go beyond the classical linear model, we opt to work with Generalized Additive Models (GAM). This allows us to infer non-linear relationships between the explained variable and the chosen explanatory variables. More precisely, a GAM expresses the expectation of $Y$ conditionally on $p$ explanatory variables $\left(X_{1}, \ldots, X_{p}\right)$, say $E\left(Y \mid X_{1}, \ldots, X_{p}\right)$, as a sum of $f_{j}\left(X_{j}\right)$

$E\left(Y \mid X_{1}, \ldots, X_{p}\right)=\sum_{j=1}^{p} f_{j}\left(X_{j}\right)+\varepsilon$,

where the function $f_{j}($.$) has a non-parametric form and \varepsilon$ represents a zero-mean Gaussian noise or error. Equation (1) justifies the term "Generalized Additive Models" because each possible explanatory variable $X_{j}$ could have a nonlinear (generalized) effect on the expectation of $Y$ and the cumulative effect of the $X_{j}$ 's is clearly additive. Although such a type of model has been extensively studied, tested and used in statistics and its related fields (Hastie and Tibshirani, 1990), it has never been applied to downscale paleoclimate data (to our knowledge).

To introduce non-linearities in (1), we chose to represent the functions $f_{j}($.$) as piecewise third order polynomial func-$ tions evaluated at four knots (i.e. borders of the "pieces" or intervals). Such functions belong to the class of cubic spline functions (e.g. de Boor, 2001). In our case, this means that each function $f_{j}($.) has at most twelve parameters (three intervals defined by four knots multiplied by four parameters for each third order polynomial). During the fitting step, the four knots are placed evenly throughout the predictor range, i.e. approximately at every 33 rd percentile of the predictor values. The values at neighbouring knots are connected by cubic polynomials constrained to continuity conditions. The latter provides smooth transitions between the different cubic polynomials at the knots. Details of the fitting methods are given in Wood $(2000,2004)$ and in the reference manual of the "mgcv" $\mathrm{R}$ package (downloadable at http://cran.r-project.org/). Note that the spline type and order were arbitrarily chosen. Our different choices (cubic splines, three intervals, continuity condition) ensure a (relatively) small number of parameters as well as enough flexibility in our modeling of transfer functions. Depending on the objectives and data at hand, other choices for the spline functions could be made.

As already mentioned, the $\varepsilon$ term of Eq. (1) represents a Gaussian error. Although temperature data classically satisfy this normality assumption, precipitation need to be log transformed before being modeled by a GAM. This is justified by the fact that cumulated precipitation can be correctly modeled by log-normal distributions (e.g. Cheng and Qi, 2002).

To illustrate the GAM capacity at modeling non-linear link functions, Fig. 1 shows the estimated spline functions $f_{j}($.) obtained after explaining some log-transformed July precipitation data with the four following explanatory variables $(p=4$ in (1)): specific humidity $(\mathrm{Q})$, wind intensity in the $\mathrm{v}$ direction (Wv), the altitude (elv), and latitude (LAT). More details about these data will be provided in Sect. 3.1. In this figure, the $\mathrm{x}$-axes correspond to each explanatory variable $X_{j}$ and the y-axes represent the contribution of $X_{j}$ at explaining $Y$, i.e. $f_{j}\left(X_{j}\right)$. The four panels indicate that the estimated contributions from $\mathrm{Q}$ and $\mathrm{Wv}$ are strongly non-linear and the ones from elv is mostly linear. The estimated $f_{j}($.$) for LAT$ is clearly uninformative since it is constantly equal to 0 . Although this first analysis was very specific and limited, it exemplifies that a GAM is very flexible and let the data speak for themselves. In particular, a non-linear relationship can be modeled only when it is necessary.

\section{Applications and results}

\subsection{The explanatory variables}

Expert knowledge leads us to select thirteen explanatory variables among all large-scale variables from our available data set. These explanatory variables are considered as potential informative candidates for explaining $E\left(Y \mid X_{1}, \ldots, X_{p}\right)$. To reflect their respective stationary quality, they have been divided into two groups. Our objective is to take into account obvious climatological facts linked to geography (e.g. elevation, continentality, slope) without an explicit physical description, and to compare them to more traditional physical 


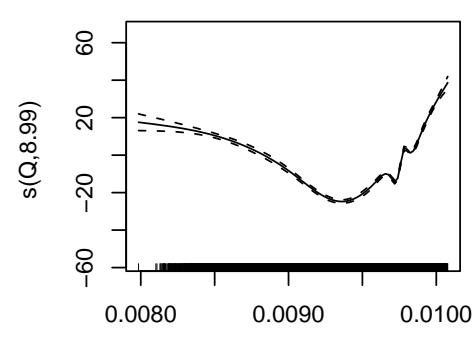

Q

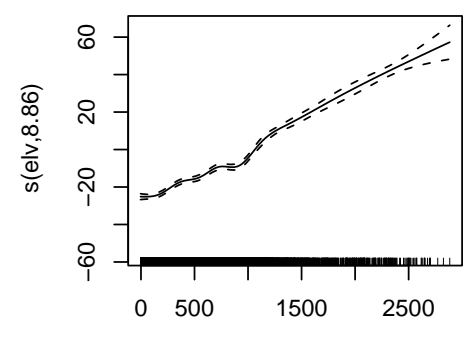

elv
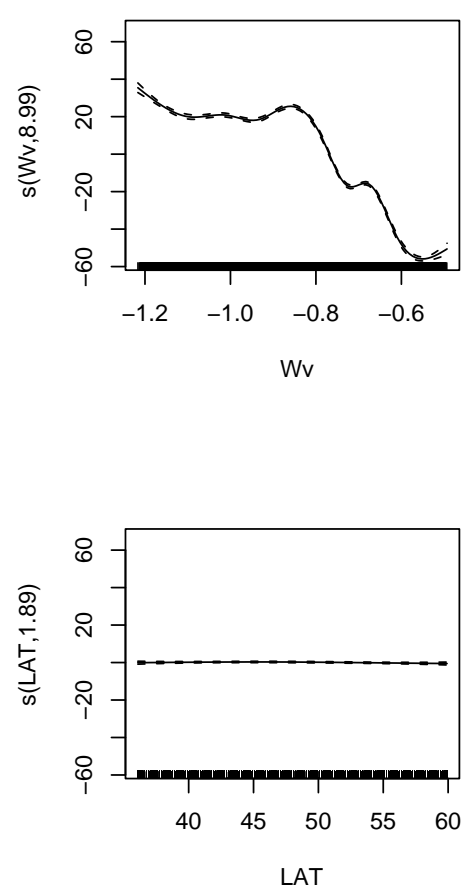

Fig. 1. Spline functions (full lines) estimated for a toy model calibrated for the July CRU precipitation data with explanatory variables Q (in $\mathrm{kg} / \mathrm{kg}$ ), Wv (in $\mathrm{m} / \mathrm{s}$ ), elv (in m), and LAT (in ${ }^{\circ} \mathrm{N}$ ). The dashed lines correspond to 2 standard errors above and below the estimated splines, and the ticks on the x-axis represent the observed predictor values. While the contributions from Q and Wv are modeled through strong non-linearity, elv and LAT contributions are mostly linear, and the LAT spline is clearly uninformative in this toy model.

predictors. For example, mountains and wind strongly influence temperature and precipitation. But modeling these influences in an explicit physical way can quickly become quite tricky. The main idea here is to let our model do this job in a statistical way, using and comparing two sets of predictors. The following nine explanatory variables are considered as "physical" variables: specific $(\mathrm{Q})$ and relative humidity (RH), sea level pressure (SLP), temperature (T), wind intensity in $\mathrm{u}(\mathrm{Wu})$ and $\mathrm{v}(\mathrm{Wv})$ directions, dew point temperature (Td), dew point temperature depression $D T d=T d-T$ and vertically integrated specific humidity (QI). In the list above, DTd represents the degree of saturation in water vapor of the atmosphere. In past studies (Charles et al., 1999; Vrac et al., 2007a; Vrac and Naveau, 2007), the variables Td and DTd have shown good explanatory power for downscaling precipitation. In addition to the "physical" variables, the following four "geographical" ones have been identified: elevation (elv), advective (Aco) and diffusive (Dco) continentality (see definition below), and W-slope (Wsl) (see definition below). The variables $\mathrm{Q}, \mathrm{RH}, \mathrm{T}, \mathrm{Wu}, \mathrm{Wv}, \mathrm{Td}, \mathrm{DTd}$, are taken at the surface.

The diffusive continentality index Dco (between 0 and 1 ) corresponds to the shortest distance to the ocean. If a point is close to a sea or an ocean, then Dco is close to zero. Conversely, a point far away from the sea translates into a Dco close to one.
The advective continentality Aco taking its value between zero and one is associated to wind intensities and directions. It is based on the following hypothesis. An air mass becomes progressively continental (or inversely maritime) as it travels over land (ocean). The rate of this changes towards continental versus maritime conditions is assumed to be a constant fraction $(\tau)$ per unit time, i.e. the change in continentality during a time $d t$ is

$d C=\left[-C\left(1-i_{c o}\right)+(1-C)\left(i_{c o}\right)\right] \tau d t$,

with $C$ the continentality ranging from zero (sea limit) to one (land limit), $i_{c o}$ equals to zero over sea and to one over land, respectively. The parameter $\tau$ satisfies $\tau d t=\tau \frac{d x}{d t} \frac{1}{U} d t=\frac{d x / U}{l_{0} / U_{0}} \ln 2$ where $d x$ is the distance traveled by the air mass during the time $d t$, $\mathrm{U}$ is the mean wind norm from CLIMBER and $l_{0} / U_{0}$ is the distance/wind ratio corresponding to a continentality change of $1 / 2$. This ratio is set to $\frac{l_{0}}{U_{0}}=\frac{5.10^{5} \mathrm{~m}}{5 \mathrm{~m} / \mathrm{s}}$. To complete the computation of continentality at a given point, we must first integrate the continentality change over each "incoming air mass path"

$C_{d}=\int_{\text {path }} d C=\int_{\text {path }}\left[-C\left(1-i_{c o}\right)+(1-C)\left(i_{c o}\right)\right] \frac{\ln (2) / U}{l_{0} / U_{0}} d x$.

It is necessary to decide the respective weight of each path direction. It is reasonable to rely on simple assumptions: (1) give more weight to path directions which matches the direction of the mean wind, and (2) give zero weight to paths 
which are in opposition with the mean wind, i.e. penalizing an air-mass traveling against the wind (this would be inconsistent with our above assumptions for the continentality change over a given path). A simple way to perform this is to use the scalar product of the mean wind $\boldsymbol{U}$ and the path direction unit vector $\hat{l}_{p}$ (integrated over each path)

$I_{d}=\int_{\text {path }} \max \left(\hat{l}_{p} \cdot \boldsymbol{U}, 0\right) d C$.

The weighted average of the contributions from all paths provides the continentality at the desired point:

$C=\frac{\sum_{d} I_{d} C_{d}}{\sum_{d} I_{d}}$.

The last "geographical" variable is the W-slope taking into account (in part) the impact of the mountains on the regional climate. It is computed separately from the advective continentality index but in a similar way. Like for continentality, several incoming air masses directions are considered, with the same weighting as before, i.e. through (4). Here, the Wslope corresponds to the mean zonal wind multiplied by the mean east-west slope over approximately $100 \mathrm{~km}$. Indeed, in CLIMBER, over this region, the prevailing wind is westerly. Only upward trends are retained. That means that the $\mathrm{W}$-slope increases only when the air mass is going up, hence potentially cooling and precipitating.

Remark that Aco and Wsl are not "purely geographical", in the sense that they are not completely static. The classification into "physical" or "geographical" predictors is (for a part) arbitrary, and reflects more "classical" predictors versus our proposed original ones, respectively.

Note also that the CLIMBER-modeled precipitation are not part of our explanatory variables set. It is common to disregard large-scale precipitation information (e.g. reanalysis data, GCM or EMIC outputs) when downscaling precipitation. Local precipitation usually have such a high spatial variability that large-scale precipitation data can be sometimes misleading (e.g., Vrac et al., 2007a).

3.2 General considerations before implementing our downscaling scheme

Spatially, one CLIMBER grid-cell corresponds to several CRU precipitation and temperature grid-cells (those contained in a CLIMBER grid-cell). This implies that our nonlinear regression scheme defined by Eq. (1) cannot be directly implemented. There are too many $Y$ 's for a single CLIMBER realization $\left(X_{1}, \ldots, X_{p}\right)$. To solve this issue, the CLIMBER outputs are bi-linearly interpolated (e.g. Accadia et al., 2003) to the CRU resolution. Hence, the interpolated CLIMBER outputs can be considered as explanatory variables (i.e., predictor values) of the CRU precipitation and temperatures in our GAM.
Concerning the seasonality, shifts in seasonality are likely to occur over long time periods and applying monthly GAMs (i.e. one GAM per month) would potentially lead to unrealistic downscaled time series. This comes back to the issue of stationary raised in Sect. 1. To overcome this problem, we restrict our attention to annual GAMs, i.e. one single model calibrated over the entire twelve months (instead of a GAM per month), meaning that the same set of spline functions is now used for all months.

We can compute the percentage of observed variance explained by a given set of explanatory variables (e.g. Saporta, 1990):

$\%$ of variance explained $=\frac{\sum_{i}\left(y_{i}^{\star}-\bar{y}\right)^{2}}{\sum_{i}\left(y_{i}-\bar{y}\right)^{2}} \times 100$

where $y_{i}^{\star}$ is the GAM-predicted value, $y_{i}$ the observed value, and $\bar{y}$ the observed mean. The values $y_{i}-y_{i}^{\star}$ (i.e. obs - prediction) are called "residuals".

In order to select the combination of explanatory variables that better explains $Y$, each possible combination among $p$ explanatory variables has been tested, plus the "null-model" corresponding to a model in which all parameters except the intercept are 0 . The number of such combinations is $N=2^{p}$. Among all combinations, the "optimal" explanatory variables set is selected according to the Bayesian Information Criterion (BIC), developed and asymptotically justified by Schwartz (1978):

$\mathrm{BIC}=n \log \left(\frac{\mathrm{RSS}}{n}\right)+d \log (n)$,

where $n$ corresponds to the sample size and the residual sum of squares (RSS) of the estimated model is defined by $\sum_{i=1}^{n}\left(y_{i}-y_{i}^{\star}\right)^{2}$. This criterion combines a term corresponding to a goodness-of-fit measure between the model and the data (the first term) with a penalty term (second term) depending on the sample size $n$ and on the dimension $d$ of the model. The BIC helps at selecting a model (and therefore the explanatory variables) by balancing the risk of over-fitting with the penalty term.

\subsection{Applying GAMs to downscaling projections}

\subsubsection{Present Western Europe downscaling projections}

Applying GAMs to all combinations of our thirteen explanatory variables clearly shows that some variables have no or very limited explicative power. For example, the spline functions obtained for $\mathrm{Wu}$ for explaining temperatures are generally very flat and close to zero (not shown). This means that although $\mathrm{Wu}$ participated to improve the percentage of variance explained, its global contribution is relatively small, of the order of the penalty term, and hence $\mathrm{Wu}$ is not essential to be kept. As explained in Sect. 3.1 and in the previous one, we define (through BIC) the "best" combinations of predictors on two different sets of variables: the "physical" and 


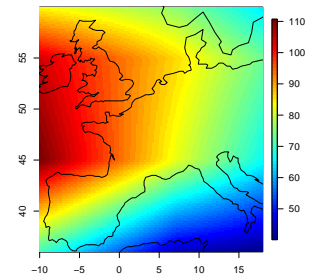

(a) Interpolated CLIMBER precipitation

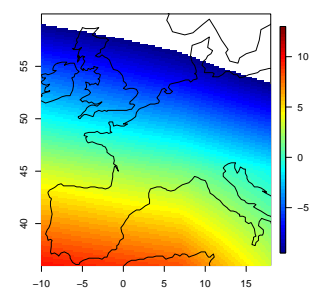

(d) Interpolated
CLIMBER tempera-
ture

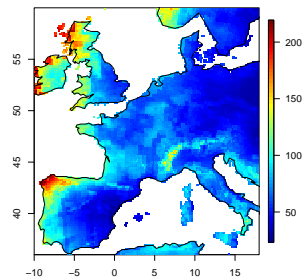

(b) GAM predicted precipitation

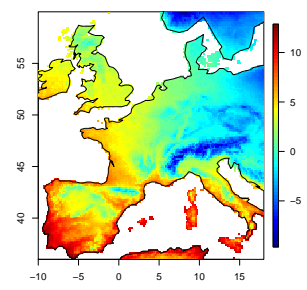

(e) GAM predicted temperature

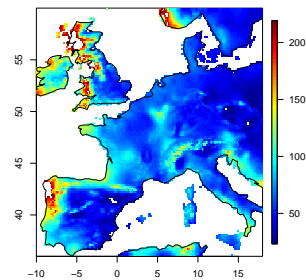

(c) Observed (CRU) precipitation

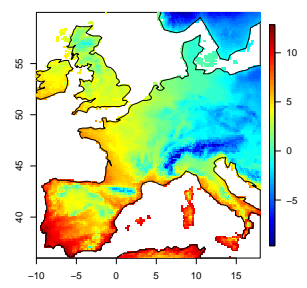

(f) Observed

(CRU) temperature

Fig. 2. January maps of (a) CRU resolution interpolated CLIMBER precipitation, (b) GAM predicted precipitation from physical variables, and (c) observed monthly precipitation (in $\mathrm{mm} /$ month). Figures (d-f) are equivalent to (a-c) but for temperatures (in ${ }^{\circ} \mathrm{C}$ ).

"geographical" ones. Overall, the final physical explanatory variables retained for our GAMs for explaining precipitation are Q, RH, T, Wu, Wv, Td, DTd, and QI. For explaining temperatures, the retained variables are slightly different: $\mathrm{Q}, \mathrm{RH}$, SLP, T, Wv, Td, DTd, and QI. These two sets of explanatory variables have the best BIC values (or second best BIC for the temperature since we removed $\mathrm{Wu}$ ) among the physical variables, and correspond to about $60 \%$ and $91 \%$ of explained variance for log-precipitation and temperatures, respectively. Concerning our "geographical" variables, the following three has been conserved to explain both temperatures and precipitation: the altitude (elv), the advective continentality (Aco) and the $\mathrm{W}$-slope (Wsl). They explain about $17.5 \%$ and $79 \%$ of variance for log-precipitation and temperatures, respectively.

In order to have a visual inspection of the quality of the results, Figure 2 displays the January maps (results are similar for the other months) of CRU resolution interpolated CLIMBER precipitation (2a), GAM predicted precipitation from the physical variables (2b), and observed monthly precipitation in $\mathrm{mm} / \mathrm{month}(2 \mathrm{c})$. Figure $2 \mathrm{~d}-\mathrm{f}$ are equivalent to $2 \mathrm{a}-\mathrm{c}$ but for temperatures $\left(\right.$ in ${ }^{\circ} \mathrm{C}$ ). The similarities between GAM-predicted and observed values are obvious and illustrate the good behavior of GAM for our Western Europe region under present climate.

Figures 3 and 4 show the residuals (obs-predicted) for four months (January, April, July, and October) representative of the four seasons, respectively for the log-precipitation with geographical explanatory variables (Figs. 3a-d) and with physical explanatory variables (Fig. 3e-h), and for the temperatures with geographical explanatory variables (Fig. 4ad) and with physical explanatory variables (Fig. 4f-i).

Although with a relatively low percentage of variance explained (about 17.5\%), the log-precipitation residuals maps obtained from the geographical variables are paradoxically small (i.e. good) and reasonably centred on zero. This low percentage of variance comes from some relatively small errors distributed over the year and by stronger residuals (meaning stronger errors) in Southern Europe in July and August. Inferred precipitation (Fig. 3) seem to be slightly better with physical variables than with geographical ones, overall for summer months. This is true also for temperatures (Fig. 4): despite systematic regional errors of about five ${ }^{\circ} \mathrm{C}$ over high mountains, physical variables seem more efficient than geographical variables to provide local predictions, i.e. with residuals closer to zero.

However, these differences between physically- and geographically-based predictions are slight and are not informative about the behaviour of the predictions in the context of a different climate. 


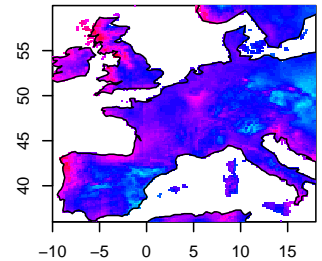

(a) January log-prec. residuals (Geo, RSS=1995)

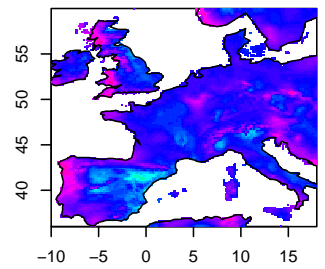

(e) January log-prec. residuals (Phy, RSS=1445)

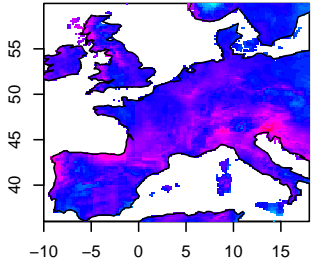

(b) April log-prec. residuals (Geo, RSS=1120)

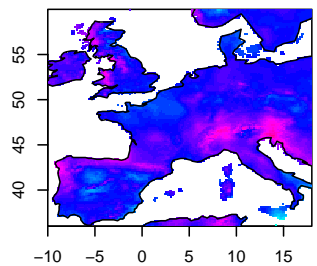

(f) April log-prec. residuals (Phy, RSS=1151)

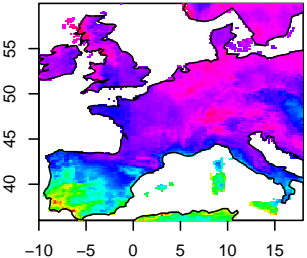

(c) July log-prec. residuals (Geo, RSS=10335)

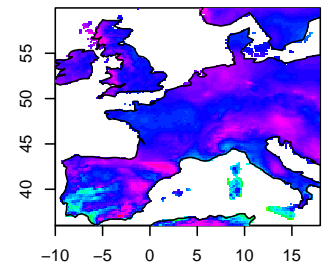

(g) July log-prec. residuals (Phy, RSS=2166)

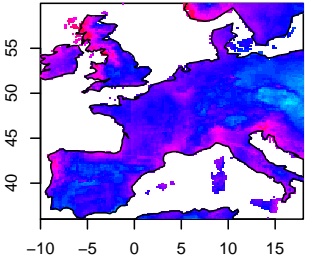

(d) October log-prec. residuals (Geo, RSS=1718)

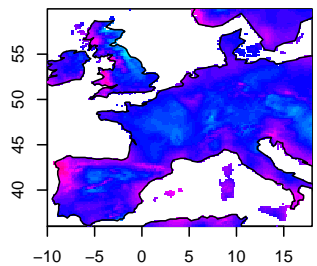

(h) October log-prec. residuals (Phy, RSS=1283)

Fig. 3. Log-precipitation GAM residuals (obs-pred) maps for Western Europe, (a-d) from the geographical explanatory variables, (e-h) from the physical explanatory variables, for January, April, July, and October respectively. The residual sum of square (RSS) is given for each month. The legend is provided in $\log (\mathrm{mm} / \mathrm{month})$ in (i).

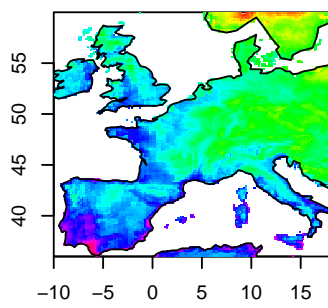

(a) January temp. residuals $(\mathrm{Geo}, \mathrm{RSS}=159973)$

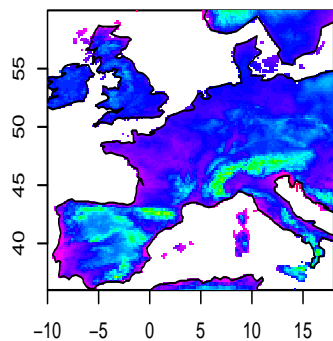

(f) January temp. residuals (Phy, RSS=46177)

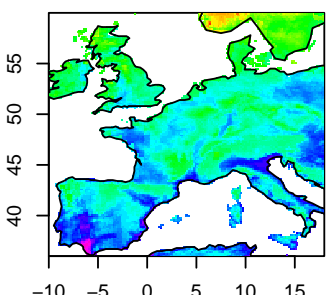

(b) April temp. residuals (Geo, RSS=95970)

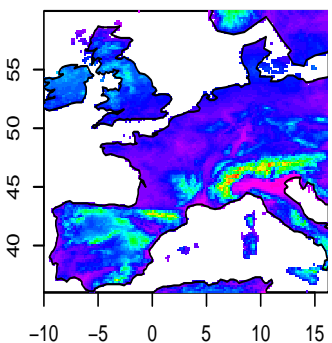

(g) April temp. residuals (Phy, RSS=62136)

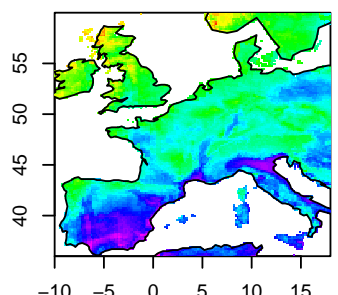

(c) July temp. residuals

(Geo, RSS=155898)

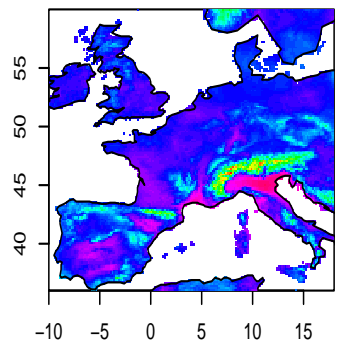

(h) July temp. residuals (Phy, RSS=51123)

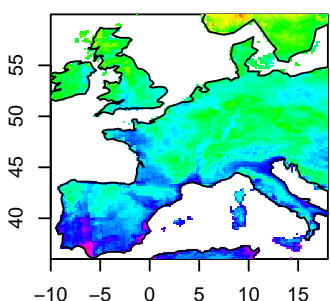

(d) October temp. residuals (Geo, RSS=109904)

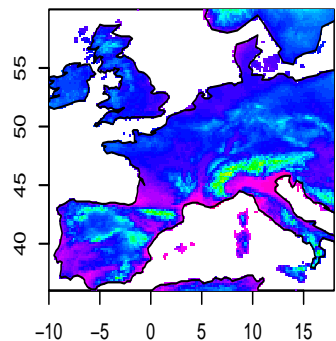

(i) October temp. residuals

(Phy, RSS=43173)

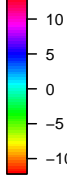

(e) Legend for $(a-d)$

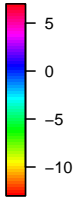

(j) Legend for (f-i)

Fig. 4. Same as Fig. 3 but for temperature. Legends are provided in ${ }^{\circ} \mathrm{C}$. 


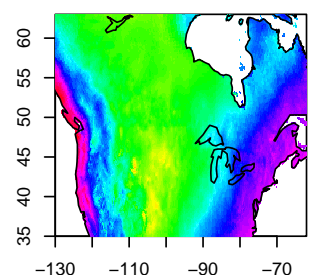

(a) January log-prec. residuals

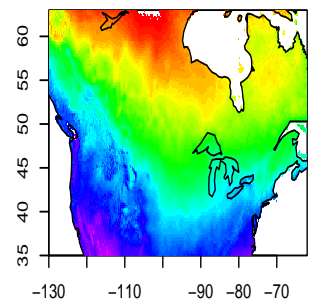

(f) January temp. residuals

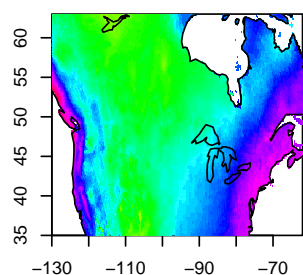

(b) April log-prec. residuals

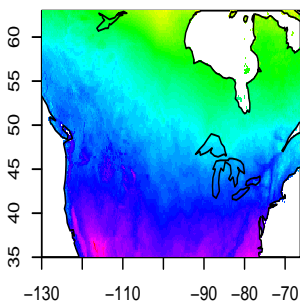

(g) April temp. residuals

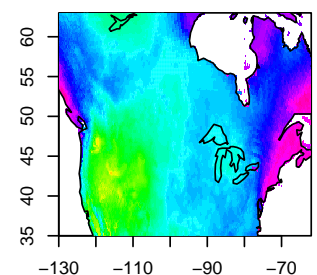

(c) July log-prec. residuals

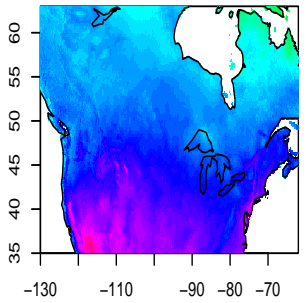

(h) July temp. residuals

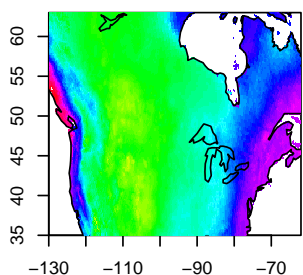

(d) October log-prec. residuals

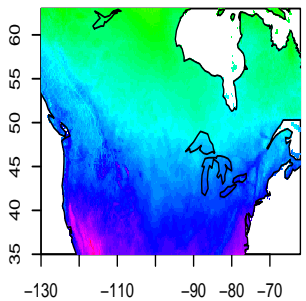

(i) October temp. residuals

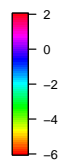

(e) Legend for $(a-d)$

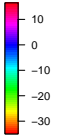

(j) Legend for $(\mathrm{f}-\mathrm{i})$

Fig. 5. (a-d) Log-precipitation and (f-i) temperature GAM residuals (obs-pred) maps for North America, from the geographical explanatory variables, for January, April, July, and October respectively. GAMs are calibrated on Western Europe. Legends are provided in $\log (\mathrm{mm} / \mathrm{month})$ in (e) and in ${ }^{\circ} \mathrm{C}$ in (j).

\subsubsection{Present downscaling projections over other regions}

In order to test the annual models for climate different from the one used for calibration, we downscale the large-scale CLIMBER data over two regions corresponding approximately to North America (USA and Canada) and Northern Europe (with a part of Siberia), based on the calibrations obtained from the two sets of predictors. The North America results of residuals (obs - pred) are presented for (log) precipitation in Fig. 5a-d and in Fig. 5f-i for temperatures, from the geographical explanatory variables. The results from the physical explanatory variables and Northern Europe results are not shown here but are discussed in the following.

North America precipitation residuals maps from the geographical variables (Fig. 5a-d) show coherent spatial structures, despite more or less pronounced residuals (from -6 to +2 units). The equivalent maps from the physical explanatory variables (not shown) are associated to larger residuals (comprised between -30 and -15 units) and to very incoherent (almost random) patterns that cannot be explained by any geographical (e.g. mountains or coasts) effects. The latter can be explained by the values of the physical explanatory variables that are out of the calibration range observed over Western Europe: for example, the range of the variable QI is $[0.4,2.4]$ for Western Europe, while it is $[0.3,3.1]$ for North America. Moreover, in Fig. 5a-d, the downscaled precipitation is associated to larger errors inland than along the East and West American coasts: the continentality index seems to play a non-negligible role. Furthermore, we can remark that the residuals are weaker for summer than for winter months.
The same conclusions hold for the precipitation projections over the Northern Europe region (not shown): residuals maps with incoherent patterns from physical explanatory variables (out of the calibration range) and with coherent spatial structures from geographical variables; weaker residuals with geographical ( -5 to +2 units) than physical variables ( 30 to -10 units); the geographical explanatory variables provide smaller errors inland than along the coasts; weaker in summer then in winter.

Figure $5 \mathrm{f}-\mathrm{i}$ for the North America temperature residuals maps show results similar to those for precipitation: incoherent maps from physical explanatory variables (not shown) with high residuals $\left(+120^{\circ} \mathrm{C}\right.$ to $\left.+220^{\circ} \mathrm{C}\right)$ and continuous aspect maps from the geographical variables with lower residuals $\left(-30^{\circ} \mathrm{C}\right.$ to $\left.+10^{\circ} \mathrm{C}\right)$, as for precipitation, smaller in summer. However, the inland/coasts differences visible for precipitation are not present for temperatures. The differences are more pronounced according to the latitudes: for example, in January, north is associated to large residuals, and the more we go south, the smaller the errors. The Northern Europe temperature results (not shown) are equivalent except for the latitudes-driven residuals. Indeed, while residuals seem to be latitudes-driven in summer, the residuals structures are longitudinal in winter (with relatively small values).

In general, the results obtained from the physical explanatory variables invalidate the Western Europe-fitted-GAM methodology when used to predict Northern Europe and North America temperatures and precipitation. Although the geographical explanatory variables alone are not entirely 


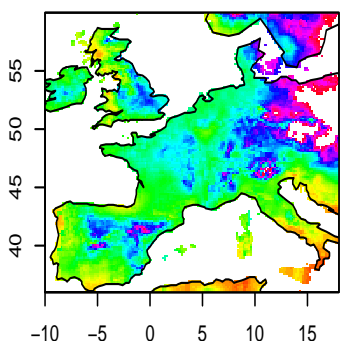

(a) January prec. relative differences

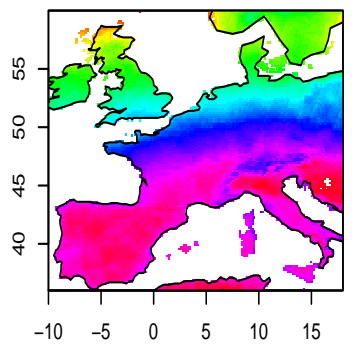

(f) January temp. absolute differences

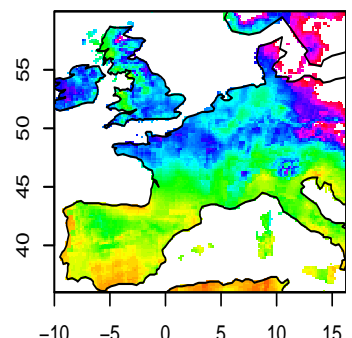

(b) April prec. relative differences

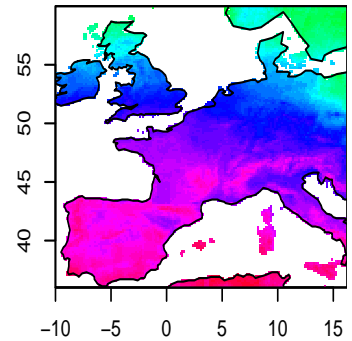

(g) April temp. absolute differences

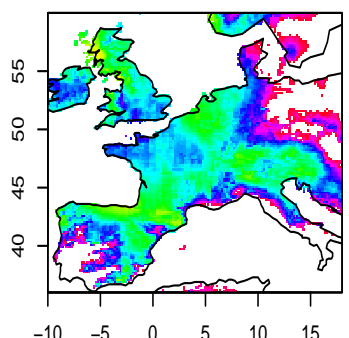

(c) July prec. relative differences

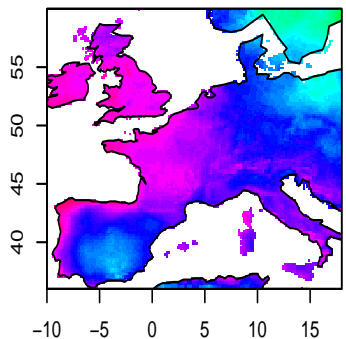

(h) July temp. absolute differences

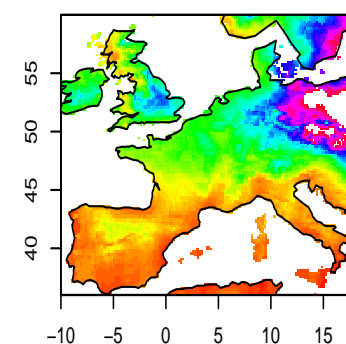

(d) October prec. relative differences

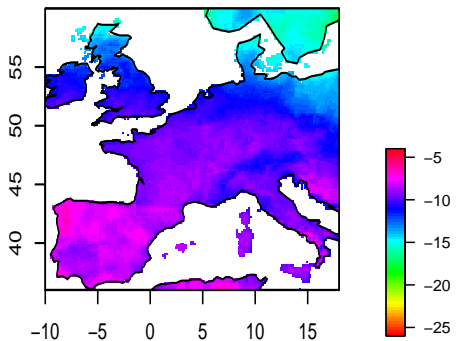

(i) October temp. absolute differences

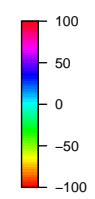

(e) Legend for $(a-d)$

(j) Legend for $(\mathrm{f}-\mathrm{i})$

Fig. 6. Monthly maps of (a-d) relative differences and (f-i) absolute differences (with respect to present) between GAM downscaled LGM values (from geographical explanatory variables and CLIMBER SLP for precipitation and from geographical explanatory variables and CLIMBER T for temperatures) and present (a-d) precipitation and (f-i) temperatures for January, April, July, and October respectively. Legends are provided in (e) in percentage and in $(\mathbf{j})$ in ${ }^{\circ} \mathrm{C}$.

satisfactory, they provide better projections (i.e. smaller residuals) than physical variables alone in downscaling precipitation and temperature values under large-scale climate conditions strongly different from the calibration ones (i.e. they are more "robust" to the change of region for projection).

\subsubsection{LGM Western Europe downscaling projections}

Based on the results brought by the projections over regions different from the calibration one, GAMs are fitted to present climate Western Europe temperatures and precipitation, and used to deduce last glacial maximum (LGM, $21 \mathrm{ky}$ ) temperatures and precipitation. For this LGM downscaling, the retained explanatory variables are the geographical variables (elv, Aco, and Wsl), where one physical predictor is added, chosen based on the BIC results and relatively subjective choices. For precipitation, this variable is the CLIMBER sea level pressure (SLP), while for temperatures it is the CLIMBER temperature $(T)$. Indeed, although the geographical explanatory variables bring robustness to the downscaling process, they are not sufficient by themselves to drive correctly the local variables. The selected added variables are supposed to provide useful large-scale information in order to have more physically-driven temperatures and precipitation. As the LGM sea level is $120 \mathrm{~m}$ lower than today, the LGM elv predictor is taken as the present altitude plus $120 \mathrm{~m}$.
Note that the ice sheets supposed to cover northern Europe are not modeled in this work, but are imposed. The splines obtained (not shown) are essentially linear and/or monotonic but not constant, confirming an underlying physical sense of the selected variables.

The monthly precipitation maps of the relative differences with respect to present precipitation are presented in Fig. 6a$\mathrm{d}$ and the monthly temperature maps of the absolute differences with respect to present temperatures are presented in Fig. 6f-i, for January, April, July, and October. Figure 6a-d show that, in general, LGM climate is drier that present one. However, regionally, some increases of the precipitation are to be noted. Moreover, the North-East part of the studied region presents a clear increase, whatever the month. For temperatures in Fig. $6 \mathrm{f}-\mathrm{i}$, as expected, we see that the LGM climate is colder than present, whatever the month and the region. A latitudinal effect is discernible from about November to May (not shown completely, see January), with stronger differences in Northern regions and smaller ones in South. LGM June-October show more uniform differences with respect to present with somehow a "coastal" effect, see for example July. These results can be quite difficult to compare to LGM GCM outputs (generally given with low spatial resolution for the whole globe). However, except for the increased rainfall in the N-E they are in a relative agreement with the LGM precipitation and temperature anomalies provided by Jost et al. (2005) for Europe. 


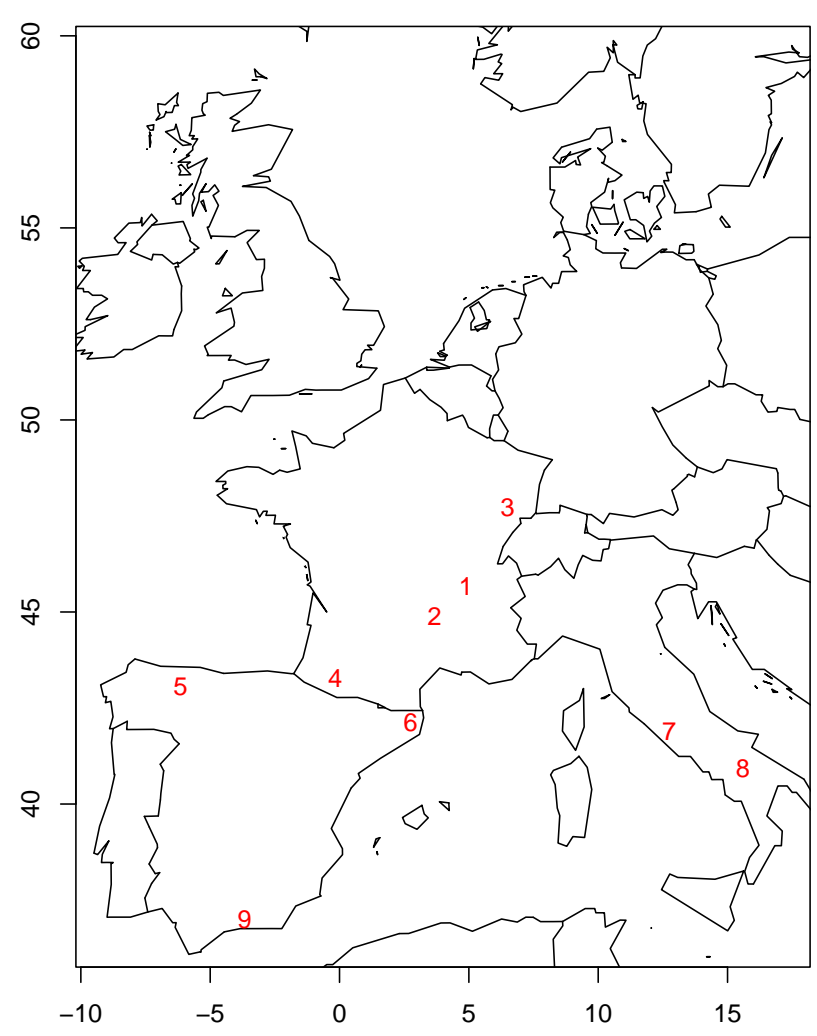

Fig. 7. Locations of the 9 reconstructed LGM data in Western Europe whose the values have to be compared to our GAM downscaled values.

In order to have more assessment of the LGM results brought by our CLIMBER-driven GAM, reconstructed LGM precipitation and temperature data have been compared to our downscaled values. These data are local reconstructions from pollen data using inverse vegetation modeling (Wu et al., 2007). The locations of the 9 points in Western Europe are shown on Fig. 7. Moreover, four GCMs involved in the Paleoclimate Modelling Intercomparison Project II (PMIP2, e.g. Braconnot et al., 2007; Kageyama et al., 2006; Ramstein et al., 2007) are also used: MIROC3.2.2, FGOALS-1.0g, IPSL-CM4-V1-MR, and HadCM3M2. These fully coupled atmosphere-ocean GCM experiments are available in the PMIP2 database as of 7 April 2007 (http://pmip2.lsce.ipsl.fr). They use the standard PMIP settings (as in CLIMBER): insolation, $\mathrm{pCO}_{2}$ and ice-sheets are prescribed. Their typical grid-size is $300 \mathrm{~km} \times 300 \mathrm{~km}$, while the CLIMBER resolution is about $1000 \mathrm{~km} \times 4000 \mathrm{~km}$. Hence, for each of the 9 locations, we have:

1. the LGM reconstructed temperature and precipitation values (min, median, and max)

2. the temperature and precipitation values for the CLIMBER grid-cells containing the points,
3. the projections obtained from the CLIMBER-driven GAM,

4. the four (temp. and precipitation) GCMs values for the model grid-cells containing the points.

For each of these 9 locations, we compare these different values for LGM January and July in Fig. 8a for temperature and in Fig. $8 \mathrm{~b}$ for precipitation. Remark that the LGM predictors values for these 9 stations do belong to the calibraton range. Note also that the CLIMBER precipitation simulations are not used in GAM for downscaling and are only provided here for comparisons.

Although the goal of this paper is not to assess the CLIMBER simulations, the most surprising result may be the good agreement between CLIMBER and the local reconstructions. For both temperature and precipitation, the GAM downscaled values are realistic and generally brought some useful additional information. Indeed, even when CLIMBER is far away from the values to be retrieved/approximated, the downscaling process is sometimes capable of moving away from CLIMBER and getting closer to the reconstructions. For example, for July precipitation (bottom of Fig. 8b), in station 9 (the right one), CLIMBER precipitation is clearly too large and the downscaled value is brought back to the low part of the GCMs range, closer to the reconstructed precipitation. A counterexample is given by stations 7 and 8 (in the same bottom of Fig. 8b), where CLIMBER precipitation is too high and the downscaled value is higher. This result is not surprising since these stations are the two locations over Italy where we see, in Fig. 6c, an unrealistic increase of precipitation larger than $100 \%$.

For temperatures, the results are generally better for January than for July. The PMIP2 GCMs used in this work give ranges of July temperatures quite far from the reconstructions. Hence, as the CLIMBER temperatures are close to the GCMs range, although the CLIMBER-driven GAM downscaled temperatures are capable of moving away from the CLIMBER values, the downscaled temperatures tend to stay distant from "real" values.

As a summary of these results, Table 1 presents the mean temperatures (in ${ }^{\circ} \mathrm{C}$ ) and mean precipitation (in $\mathrm{mm} /$ month) computed for January and July for the 9 stations from the reconstructed data, the GCMs outputs, the bi-linearly interpolated CLIMBER values, and the GAM projections. We see that the mean GAM downscaled values are closer to the reconstructions than CLIMBER and the PMIP2 GCMs for July temperatures and January precipitation, while CLIMBER is the closest to reconstructions for January temperatures (with GAM projections better than the GCM's), and the GCMs are the closest to the reconstructed July precipitation. This last point is due to stations 7 and 8 with too large downscaled values for the July mean precipitation. However, in general, the CLIMBER-driven GAM based downscaling process provides realistic and satisfying local temperatures and precipitation, thus showing the quality of the proposed method. 

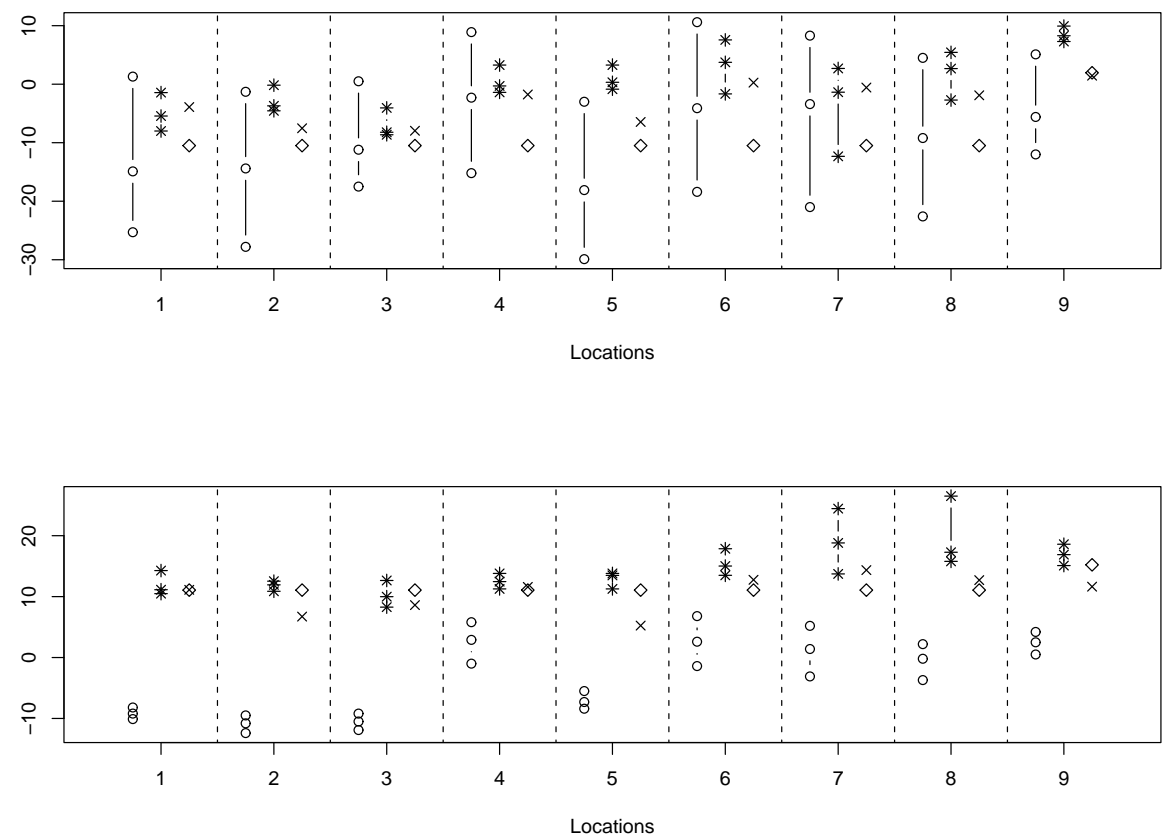

(a) January (top) and July (bottom) temperatures
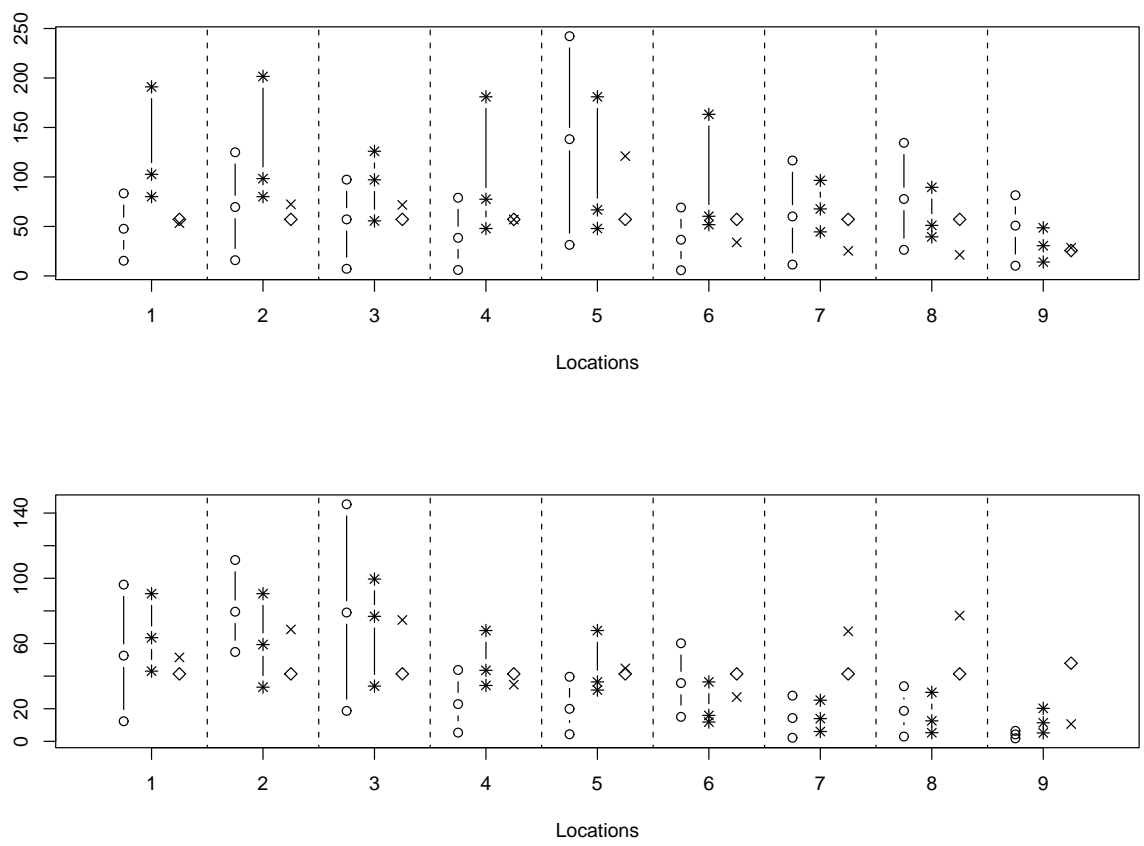

(b) January (top) and July (bottom) precipitation

Fig. 8. (a) Comparison (in ${ }^{\circ} \mathrm{C}$ ) at the 9 locations of the reconstructed temperatures (min, median, and max in o signs), the min, median, and max temperature values (in asterisk signs) from the four GCMs grid-cell (containing the location), the CLIMBER grid-cell (containing the location) temperatures (in diamond signs), and the CLIMBER-driven GAM downscaled temperatures (in $\times$ signs) for January and July ; (b) idem for precipitation values (in $\mathrm{mm} / \mathrm{month}$ ). 
Table 1. Mean temperatures (in ${ }^{\circ} \mathrm{C}$ ) and precipitation (in $\mathrm{mm} / \mathrm{month}$ ) computed for January and July from the 9 locations. The ${ }^{\star}$ symbol indicates the value the closest to the reconstructions.

\begin{tabular}{lll}
\hline January mean temperatures & from reconstructions & -9.2 \\
& from GCMs & -0.4 \\
& from CLIMBER & $-9.1^{\star}$ \\
& from GAM & -3.2 \\
July mean temperatures & from reconstructions & -3.2 \\
& from GCMs & 14.4 \\
& from CLIMBER & 11.5 \\
& from GAM & $10.5^{\star}$ \\
January mean precipitation & from reconstructions & 64 \\
& from GCMs & 84.5 \\
& from CLIMBER & 53.6 \\
July mean precipitation & from GAM & $53.8^{\star}$ \\
& from reconstructions & 36.3 \\
& from GCMs & $38.9^{\star}$ \\
& from CLIMBER & 42.1 \\
& from GAM & 49.7 \\
\hline
\end{tabular}

\section{Conclusions and discussion}

In this article, Generalized Additive Models have been developed to inject non-linearities in the downscaling of precipitation and temperatures within a paleoclimate framework (LGM and present). Two types of explanatory variables, called "geographical" and "physical", have been investigated in our non-linear regression scheme. Our analysis tends to show that the geographical explanatory variables alone are not entirely satisfactory, but provide more "realistic" local projections than physical variables alone. If used alone, the latter seem to be uninformative at best and misleading at worst. This is particularly true when they are exploited outside of their calibration range (e.g., transfer functions trained on Western Europe and applied to North America). In contrast, the geographical ones improve the stability of our downscaling projections.

After calibration on present climate, selected geographical variables (altitude, advective continentality, and W-slope) associated with physical ones (temperatures and SLP from the CLIMBER model), local temperature and log-transformed precipitation projections at the LGM were derived from our GAMs. The resulting downscaled maps showed realistic decreases in temperature and precipitation with respect to present climate, at least over France, i.e. the central part of the Western Europe region. Compared to LGM reconstructions of temperatures and precipitation and simulations from four GCMs involved in PMIP2, our downscaled values behave reasonably well at least for July temperatures and January precipitation.

As a technical perspective, it could be interesting to study how to give less liberty to the functions of our statistical model, in order to avoid too much influence from details spe- cific to the climate of a given region or time period. This could be done by verifying to what extend any part of the modeled transfer functions are physically interpretable (or at least compatible with our actual understanding of climate).

As a more general perspective, it would be interesting to generate continuous long time series (e.g. thousand years or more) of local temperatures and precipitation by taking advantage of the couple CLIMBER/GAM. The produced data could help our understanding of the past climate evolution by comparing the generated time series with existing reconstructions.

It would also be worth calibrating and applying the GAM approach to the GCMs involved in PMIP2 instead of the CLIMBER model. Indeed, although adaptations (in the predictors and/or in GAM) have probably to be made in this context, as GCMs represent the atmospheric dynamics at much higher spatial and temporal resolutions than CLIMBER, sharper downscaling results should be obtained. In addition, one can also increase the number of EMIC or GCM runs. Ensemble techniques should improve the estimation of the variability within our downscaling procedure.

Acknowledgements. Although this work has been funded in part by the Agence Nationale pour la gestion des Déchets RAdioactifs (ANDRA), it has not been subjected to the Agency's required peer and policy review and therefore does not necessarily reflect the views of the Agency, and no official endorsement should be inferred.

We acknowledge the international modeling groups for providing their data for analysis, the Laboratoire des Sciences du Climat et de l'Environnement (LSCE) for collecting and archiving the model data. We also acknowledge $\mathrm{H}$. Wu for providing the reconstruction data.

The PMIP2/MOTIF Data Archive is supported by CEA, CNRS, the EU project MOTIF (EVK2-CT-2002-00153) and the Programme National d'Etude de la Dynamique du Climat (PNEDC). The analyses were performed using version 06-04-2007 of the database. More information is available on http://www-1sce.cea.fr/pmip/ and http://www-lsce.cea.fr/motif/.

This work was supported by the European E2-C2 grant, the National Science Foundation (grant: NSF-CMG (ATM-0327936)) and the ANR-AssimilEx project.

The authors would also like to credit the contributors of the $\mathrm{R}$ project. The GAM fitting has been realized through the $\mathrm{R}$ software with the package "mgcv" downloadable on the $\mathrm{R}$ project for statistical computing website (http://www.r-project.org/).

Edited by: M.-F. Loutre

\section{References}

Accadia, C., Mariani, S., Casaioli, M, Lavagnini, A., and Speranza, A.: Sensitivity of Precipitation Forecast Skill Scores to Bilinear Interpolation and a Simple Nearest-Neighbor Average Method on High-Resolution Verification Grids, Weather Forecast., 18, 918-932, 2003. 
Bardossy, A., Muster, H., Duckstein, L., and Bogardi, I.: Automatic classification of circulation patterns for stochastic precipitation modelling. Stochastic and Statistical Methods in Hydrology and Environmental Engineering, 1. Extreme Values: Floods and Droughts, 1994.

Berger, A.: Long-term variations of daily insolation and quaternary climatic changes, J. Atmos. Sci., 35, 2362-2367, 1978.

de Boor, C.: A Practical Guide to Splines. Springer Series: Applied Mathematical Sciences, Vol. 27 1st ed. 1978, 1st hardcover printing, XVIII, 372 p. ISBN: 978-0-387-95366-3, 2001.

Braconnot, P., Otto-Bliesner, B., Harrison, S., Joussaume, S., Peterschmitt, J.-Y., Abe-Ouchi, A., Crucifix, M., Driesschaert, E., Fichefet, Th., Hewitt, C. D., Kageyama, M., Kitoh, A., Laîné, A., Loutre, M.-F., Marti, O., Merkel, U., Ramstein, G., Valdes, P., Weber, S. L., Yu, Y., and Zhao, Y.: Results of PMIP2 coupled simulations of the Mid-Holocene and Last Glacial Maximum Part 1: experiments and large-scale features, Clim. Past, 3, 261277, 2007, http://www.clim-past.net/3/261/2007/.

Charles, S. P., Bates, B. C., Whetton, P. H., and Hughes, J. P.: Validation of downscaling models for changed climate conditions: case study of southern Australia, Clim. Res., 12, 1-14, 1999.

Cheng, M. and Qi, Y.: Frontal Rainfall-Rate Distribution and Some Conclusions on the Threshold Method, J. Appl. Meteorol., 41, 1128-1139, 2002.

Hastie, T. J. and Tibshirani, R. J.: Generalized Additive Models, Chapman and Hall, 1990.

Huth, R.: Disaggregating climatic trends by classification of circulation patterns, Int. J. Climatol., 21, 135-153, 2001.

Huth, R.: Statistical downscaling of daily temperature in central Europe, J. Climate, 15, 1731-1742, 2002.

Jost, A., Lunt, D., Kageyama, M., Abe-Ouchi, A., Peyron, O., Valdes, P.J., and Ramstein, G.: High-resolution simulations of the last glacial maximum climate over Europe: a solution to discrepancies with continental palaeoclimatic reconstructions?, Clim. Dynam., 24, 577-590, 2005.

Kageyama, M., Peyron, O., Pinot, S., Tarasov, P., Guiot, J., Joussaume, S., and Ramstein, G.: The Last Glacial Maximum climate over Europe and western Siberia: a PMIP comparison between models and data, Clim. Dynam., 17, 23-43, 2001.

Kageyama, M., Laîné, A., Abe-Ouchi, A., Braconnot, P., Cortijo, E., Crucifix, M., de Vernal, A., Guiot, J., Hewitt, C. D., Kitoh, A., Kucera, M., Marti, O., Ohgaito, R., Otto-Bliesner, B., Peltier, W. R., Rosell-Melé, A., Vettoretti, G., Weber, S. L., and Yu, Y.: MARGO Project Members. Last Glacial Maximum temperatures over the North Atlantic, Europe and western Siberia: a comparison between PMIP models, MARGO sea-surface temperatures and pollen-based reconstructions, Quat. Sci. Rev., 25, 2082-2102, 2006.

Katz, R. W.: Techniques for estimating uncertainties in climate change scenarios and impact studies, Clim. Res., 20, 167-185, 2002.

Liang, X.-Z., Pan, J., Zhu, J., Kunkel, K. E., Wang, J. X. L., and Dai, A.: Regional climate model downscaling of the u.s. summer climate and future change, J. Geophys. Res., 111, D10108, doi:101029/2005JD006685, 2006.

New, M., Lister, D., Hulme, M., and Makin, I.: A high-resolution data set of surface climate over global land areas, Clim. Res., 21, $1-25,2002$.
Peltier, W. R.: Ice age paleotopography, Science, 265, 195-201, 1994.

Petit, J. R., Jouzel, J., Raynaud, D., Barkov, N. I., Barnola, J.-M., basile, I., bender, M., Chappelaz, J., Davis, M., Delaygue, G., Delmotte, M., Kotlyakov, V. M., legrand, M., Lipenkov, V. Y., Lorius, C., Ppin, L., Ritz, C., Saltzman, E., and Stievenard, M.: Climate and atmospheric history of the past 420000 years from the Vostok ice core, Antarctica, Nature, 399, 429-436, 1999.

Petoukhov, V., Ganopolski, A., Brovkin, V., Claussen, M., Eliseev, A., Kubatzki, C., and Rahmstorf, S.: CLIMBER-2: a climate system model of intermediate complexity, Clim. Dynam., 16, 117, 2000.

Petoukhov, V., Claussen, M., Berger, A., Crucifix, M., Eby, M., Eliseev, A. V., Fichefet, T., Ganopolski, A., Goosse, H., Kamenkovich, I., Mokhov, I. I., Montoya, M., Mysak, L. A., Sokolov, A., Stone, P., Wang, Z., and Weaver, A. J.: EMIC Intercomparison Project (EMIP-CO2): comparative analysis of EMIC simulations of climate, and of equilibrium and transient responses to atmospheric CO2 doubling, Clim. Dynam., 25, 363385, 2005.

Rahmstorf, S. and Ganopolski, A.: Long-Term Global Warming Scenarios Computed with an Efficient Coupled Climate Model, Climatic Change, 43, 353-367, 1999.

Ramstein, G., Kageyama, M., Guiot, J., Wu, H., Hély, C., Krinner, G., and Brewer, S.: How cold was Europe at the Last Glacial Maximum? A synthesis of the progress achieved since the first PMIP model-data comparison, Clim. Past. Discuss., 3, 1-24, 2007.

Saporta, G.: Probabilités, Analyse des données et statistiques, Editions Technip, 1990.

Schwartz, G.: Estimating the dimension of a model, The Annals of Statistics, 6, 461-464, 1978.

Snell, S. E., Gopal, S., and Kaufmann, R. K.: Spatial interpolation of surface air temperatures using artificial neural networks: Evaluating their use for downscaling GCMs, J. Climate, 13, 886-895, 2000.

Vrac, M. and Naveau, P.: Stochastic downscaling of precipitation: From dry events to heavy rainfalls, Water Resour. Res., 43, W07402, doi:10.1029/2006WR005308, 2007.

Vrac, M., Stein, M., and Hayhoe, K.: Statistical downscaling of precipitation through a non-homogeneous stochastic weather typing approach, Climate Res., 34, 169-184, doi:10.3354/cr00696, 2007a.

Vrac, M., Stein, M., Hayhoe, K., and Liang, X. L.: A general method for validating statistical downscaling methods under future climate change, Geophys. Res. Lett., 34, L18701, doi:10.1029/2007GL030295, 2007b.

Wilby, R. L., Wigley, T. M. L., Conway, D., Jones, P. D., Hewitson, B. C., Main, J., and Wilks, D. S.: Statistical downscaling of general circulation model output: A comparison of methods, Water Resour. Res., 34, 2995-3008, 1998.

Wilks D. S. and Wilby, R. L.: The weather generation game: a review of stochastic weather models, Progress in Physical Geography, 23, 329-357, 1999.

Wilks, D. S.: Multisite downscaling of daily precipitation with a stochastic weather generator, Clim. Res., 11, 125-136, 1999.

Wood, A. W., Leung, L. R., SRidhar, V., and Lettenmaier, D. P.: Hydrologic implications of dynamical and statistical approaches to downscaling climate model outputs, Clim. Change, 62, 189- 
216, 2004

Wood, S. N.: Modelling and Smoothing Parameter Estimation with Multiple Quadratic Penalties, J. R. Statist. Soc. B, 62(2), 413428, 2000.

Wood, S. N.: Stable and efficient multiple smoothing parameter estimation for generalized additive models, J. Amer. Statist. Ass., 99, 673-686, 2004.
Wu, H., Guiot, J., Brewer, S., and Guo, Z.: Climatic changes in Eurasia and Africa at the last glacial maximum and mid-Holocene: reconstruction from pollen data using inverse vegetation modelling, Clim. Dynam., 29, 211-229, doi:10.1007/s00382-007-0231-3, 2007.

Zorita, E. and von Storch, H.: The analog method as a simple statistical downscaling technique: Comparison with more complicated methods, J. Climate, 12, 2474-2489, 1998. 